\title{
General triple charged black ring solution in supergravity
}

\section{Andrew Feldman and Andrei A. Pomeransky}

Budker Institute of Nuclear Physics,

11, academician Lavrentiev Ave., Novosibirsk, Russia

Physics Department, Novosibirsk State University,

2, Pirogova Str., Novosibirsk, Russia

E-mail: andrew.1.feldman@gmail.com, pomeransky@gmail.com

ABSTRACT: We present the general black ring solution in $\mathrm{U}(1)^{3}$ supergravity in 5 dimensions with three independent dipole and electric charges. This immediately gives the general black ring solution in the minimal 5D supergravity as well.

Keywords: Classical Theories of Gravity, Black Holes, Black Holes in String Theory, Supergravity Models

ArXiv EPrint: $1406.4617 \mathrm{v} 1$ 


\section{Contents}

1 Introduction 1

2 Notations 2

3 Derivation 4

4 Solution 5

$\begin{array}{lll}5 & \text { Conclusions } & 6\end{array}$

$\begin{array}{lll}\text { A Field equations } & 7\end{array}$

$\begin{array}{lr}\text { B Set of functions } & 8\end{array}$

\section{Introduction}

Black rings are a type of black hole solutions in 5-dimensional spacetime with the event horizon homeomorphic to $S^{1} \times S^{2} \times R$ (see [1] for a review). After the discovery of neutral black rings in pure gravity [2] the charged generalizations in supergravity were considered soon [3-6]. While the most general supersymmetric solution was found [7-10], the known families of nonextremal solutions lacked a number of free parameters. In this paper we present for the first time the general nonextremal solution in $\mathrm{U}(1)^{3}$ supergravity. The solution has three independent electric and dipole charge parameters, plus 5 additional independent parameters. The general solution has the usual Dirac-Misner string and conical singularities. The condition of the absence of singularities then singles out the family of regular solutions with the complete set of independent parameters: 3 electric charges, 3 dipoles, 2 angular momenta and mass. As is well known, the corresponding solutions of the 5D minimal supergravity can be immediately obtained by setting the three electric charges and the three dipole charges equal, which makes all three gauge fields equal and all dilatons constant.

Our starting point is the solution with a single nonzero gauge field that was found in [11-13] using the inverse scattering method [14-16]. It was pointed out in [17] that when 2 gauge fields and a dilaton of $\mathrm{U}(1)^{3}$ supergravity are set equal to zero, the result is the 5D Kaluza-Klein theory: the pure 6-dimensional gravity compactified on a circle. And therefore, it was possible to apply to this $6 \mathrm{D}$ pure gravity theory the procedure outlined in [18] based on the inverse scattering method and used in [19] to derive the general black ring solution in the 5D pure gravity. In the concluding section of [13] we suggested that the missing parameters can be introduced into the solution by symmetrizing it with respect to the dipole charges. The idea is that the general solution should be invariant under the 
simultaneous permutations of the gauge fields, dilatons and corresponding dipole charges. The observed lack of symmetry is the consequence of 2 dipole charges parameters being set to some particular value and when one eliminates this asymmetry one gets the general solution. This turned out to be indeed possible to do, but first one needs to eliminate another source of asymmetry coming from a somewhat arbitrary choice of coordinates used in [13]. We explain the details of this derivation in section 3 after introducing various necessary notations in section 2. The solution is presented in section 4 using a set of functions introduced in the appendix B. As we have checked numerically with the precision better than $10^{-100}$ the solution indeed satisfies the field equations written down in the appendix A. In the concluding section 5 we discuss some possible directions for the future work.

\section{Notations}

Let us start by introducing the necessary notations for coordinates, parameters, fields and so on. The metric components, as well as all other fields, depend only on two coordinates: $u$ and $v$. There are also three other coordinates in the 5D space-time: $t, \phi, \psi$ of which the metric is independent. They correspond therefore to three commuting symmetries that can be described by three commuting Killing vectors. There is also a not so short list of parameters. First of all, the four parameters $x_{i}, i=0,1,2,3$ give positions of poles in the inverse metric. These poles are related to the very useful notion of rods [20]. By a Möbius transformation of coordinates $u$ and $v$ one can take three of the $x_{i}$ to the arbitrary values, while only the remaining fourth constant is indeed a parameter. A popular choice is $x_{1}=-1, x_{2}=1, x_{3}=-1 / c, x_{0}=\infty$. Then there are three parameters $a_{i}$, $i=1,2,3$ that are needed to obtain by imposing the necessary regularity conditions the general nonsingular doubly rotating solution. Furthermore, there are four parameters $y_{i}$, $i=0,1,2,3$. Three of them are related to dipole charges, but $y_{0}$ is already present in the Emparan-Reall neutral black ring with a single rotation. Finally, one can apply a sequence of three boosts, interspersed with duality transformations, to charge the solution with respect to three independent electric charges. It is convenient to characterize the boosts by their velocities $\beta_{i}, i=1,2,3$.

Despite the large number of parameters it is possible to present the solution in a relatively compact and readable form thanks to its numerous symmetries. Let us consider a set of three transformations $(i=1,2,3)$ :

$$
u \rightarrow h_{i}(v), v \rightarrow h_{i}(u), a_{i} \rightarrow 1 / a_{i}
$$

Here $h_{1}(u)$ is the Möbius transformation with the properties: $h_{1}\left(x_{1}\right)=x_{0}, h_{1}\left(x_{2}\right)=x_{3}$ and $h_{1}\left(h_{1}(u)\right)=u$. The explicit expression is

$$
h_{1}(u)=\frac{\left(x_{2} x_{3}-x_{0} x_{1}\right) u+x_{0} x_{1}\left(x_{2}+x_{3}\right)-x_{2} x_{3}\left(x_{0}+x_{1}\right)}{\left(x_{2}+x_{3}-x_{0}-x_{1}\right) u+x_{0} x_{1}-x_{2} x_{3}} .
$$

$h_{2}(u)$ and $h_{3}(u)$ can be obtained from $h_{1}(u)$ by exchanging $x_{1}$ with $x_{2}$ and with $x_{3}$, respectively. All components of all fields (metric, gauge fields and scalars) of the solution do not change under the transformations 2.1. In turn, this invariance follows from the fact 
that all these components have the form of ratios, and under the discussed transformation numerators and denominators of the ratios are multiplied by the same common factor. The numerators and denominators are polynomials in each $a_{i}$ of degree at most 2 . The invariance under 2.1 allows one to express the coefficient of $a_{i}^{2}$ in these polynomials in terms of the term of zero degree in $a_{i}$. To this end we introduce the symmetrization operators $S_{i}$, which act in the space of functions of $u$ and $v$ as follows:

$$
S_{i}\{f(u, v)\}=f(u, v)-a_{i}^{2} f\left(h_{i}(v), h_{i}(u)\right) .
$$

The operators $S_{i}$ commute with each other. The composition of all $S_{i}$ will be denoted by $S\{f\}=S_{1}\left\{S_{2}\left\{S_{3}\{f\}\right\}\right\}$.

We never use summation over repeating indices, except when the sum is written explicitly. We use the convention that the indices $i, j, k, l$ have arbitrary but different values, in other words they represent a permutation of $(0,1,2,3)$. We will use frequently 6-component quantities, where each component corresponds to an unordered pair of indices $i$ and $j(i \neq j)$. The addition and multiplication for them is the component-wise one. The double vertical line brackets $\| \ldots$. $\|$ will denote the sum of all 6 components of the quantity in the brackets. We introduce two functions of a single variable $l(z)$ and $q(z)$ that take such 6-component values:

$$
l_{i j}(z)=\left(z-x_{i}\right)\left(z-x_{j}\right), q_{i j}(z)=\sqrt{z-x_{i}} \sqrt{z-x_{j}},
$$

and a 6-component valued function of two variables $r(u, v)$ :

$$
r_{i j}(u, v)=\frac{\left(u-x_{i}\right)\left(v-x_{i}\right)}{G^{\prime}\left(x_{i}\right)}+\frac{\left(u-x_{j}\right)\left(v-x_{j}\right)}{G^{\prime}\left(x_{j}\right)},
$$

where

$$
G(u)=\prod_{i=0}^{3}\left(u-x_{i}\right) .
$$

Let us introduce also a constant 6-component quantity $\Delta$ with the components equal to $\Delta_{i j}=\left(x_{i}-x_{j}\right)^{2}$. For a 6 -component quantity we will denote by bar the transposition, which consists in exchanging $i j$ with $k l$ components, for example: $\bar{q}_{i j}=q_{k l}$. Note that $r_{i j}(u, v)=-r_{k l}(u, v)$, or with the above notation: $\bar{r}(u, v)=-r(u, v)$. It is also useful to combine the constants $a_{i}$ into the 6-component constant $a$ with the components $a_{0 i}=a_{i}$, and the property $\bar{a}=-a$. Let us introduce also a trilinear function of 3 variables $c(p, s, t)$, where each of the variables $s, p$ and $t$ is a 6 -component quantity, and the function is equal to

$$
c(p, s, t)=\frac{1}{2} \sum_{i \neq j \neq k} p_{i j} s_{j k} t_{k i},
$$

where the sum is over all 24 ordered triplets $(i, j, k)$ of non-equal values of indices. Such function corresponds to a totally symmetric tensor of rank 3 . When one takes all three arguments equal, one obtains a cubic function that we will call $c_{3}: c_{3}(t)=\frac{1}{3} c(t, t, t)$. We will use also a symmetrization operator $S^{\prime}$, which acts on the 6-component quantities as follows:

$$
S^{\prime}(t)_{0 i}=S_{j}\left(S_{k}\left(t_{0 i}\right)\right), S^{\prime}(t)_{i j}=S_{i}\left(S_{j}\left(t_{i j}\right)\right),
$$

where the triple $\{i, j, k\}$ is a permutation of $\{1,2,3\}$. 
Having introduced all these notations, we are now ready to write down the complete set of functions of coordinates and parameters which appear in the solution. This is done in the appendix B. This set is comprised of ten functions $H_{i j}, K_{i j}, \Omega^{i j}, \omega_{i}, \Sigma_{i}, \Pi_{i j}, \Xi_{i j}, Q$, $Z$, and $g^{\varphi \psi}$.

\section{Derivation}

Let us now explain how we were able to recover the general family of solutions which is the subject of this paper, starting from its particular subfamily presented in our previous paper [13]. The solution in [13] depends among others on 2 parameters $y_{1}$ and $y_{2}$. It will be convenient to shift the index and rename the parameters $y_{0}$ and $y_{1}$. Recall also, that in [13] we had the following choice of parameters that specify the positions of poles in the inverse metric: $x_{i}$ for $i=1,2,3$ in arbitrary positions, and the fourth pole fixed at the infinity: $x_{0}=\infty$. If one wants to put the solution in the maximally symmetric form, one would like to treat all $x_{i}$ on equal footing and make $x_{0}$ a free parameter too. This can be done by a coordinate transformation, making substitutions $u \rightarrow h(u)$ and $v \rightarrow h(v)$, where $h(v)$ is a Möbius transformation that has a pole at the point $x_{0}$ :

$$
h(u)=\frac{\alpha u+\beta}{u-x_{0}} .
$$

One has to make also the same substitution for the constants: $x_{i} \rightarrow h\left(x_{i}\right), i=1,2,3$ and $y_{i} \rightarrow h\left(y_{i}\right)$. At the same time one rescales the constants

$$
a_{i} \rightarrow a_{i} \frac{\beta+\alpha x_{0}}{x_{i}-x_{0}} \frac{\sqrt{x_{i}-y_{0}} \sqrt{x_{i}-y_{1}}}{\sqrt{x_{0}-y_{0}} \sqrt{x_{0}-y_{1}}}
$$

$i=1,2,3$ in order to eliminate everywhere the dependence on $\alpha$ and $\beta$ and to put $x_{0}$ on equal footing with other $x_{i}$.

In the new coordinates the solution has the following instructive property. Let us take $g^{\varphi \varphi}$ component as the simplest example. It contains a term with the following product $\left(u-x_{0}\right)^{2}\left(u-y_{0}\right)\left(u-y_{1}\right)$. One can say that the explicit dependence on $x_{0}$, which violates the symmetry among all $x_{i}$, is the effect of setting $y_{2}=y_{3}=x_{0}$ in the symmetric general solution. We see that if one replaces $\left(u-x_{0}\right)^{2}$ by $\left(u-y_{2}\right)\left(u-y_{3}\right)$, one restores both symmetry and generality (hopefully) at the same time. We have applied this idea systematically to all components of inverse metric, gauge fields and scalars. A minor complication is that not all components should be symmetric in all $y_{i}$. Only $g^{\varphi \varphi}, g^{\psi \psi}$ and $g^{\varphi \psi}$ have this total symmetry. Other components of the inverse metric, namely $g^{t t}, g^{t \varphi}$ and $g^{t \psi}$, single out $y_{0}$ but should be symmetric in $y_{1}, y_{2}$ and $y_{3}$. The gauge fields $A_{j}$ single out the corresponding $y_{j}$ and $y_{0}$, but should be symmetric in the remaining two $y_{i}(i \neq j \neq 0)$. The same is true for scalar fields. After one gains some experience, the symmetrization procedure becomes almost straightforward, only with a small amount of guesswork needed. Fortunately, there is an excellent way to check the correctness for each component of the inverse metric separately: the residues at the poles in $u$ should not depend on $v$ apart from a common factor which is easy to cancel completely. Furthermore, the residues should factorize: $\operatorname{res}\left(g^{i j}\right) \sim \rho^{i} \rho^{j}$, 
where $\rho^{i}$ is a constant vector — the rod direction (for the description of rods see [20]). This test is very strict because it is extremely improbable to have such factorization to hold by chance in an incorrect expression. When all components of the inverse metric have been obtained, one more test becomes available: it turns out that the determinant of the $3 \times 3$ matrix of inverse metric components for coordinates $t, \varphi$ and $\psi$ has a very simple form $\operatorname{det}\left(g^{i j}\right)=\frac{(u-v)^{4}}{G(u) G(v)}$. After all fields have been found, one can finally check that they indeed satisfy the field equations. Due to the high enough complexity of the solution, we were not able to perform this check analytically even with the help of a computer algebra system. Instead, it was possible to do this numerically with a precision of more than 100 digits. Such numerical precision is absolutely sufficient to convince everyone that the solution is correct. We used Wolfram Mathematica for both algebraic manipulations and numerical calculations. A Mathematica notebook that contains the solution and the numerical checks of the field equations is available on request.

Once the solution with general values of the dipole charges is found one can turn on the electric charges too. There is a well-known procedure for charging a $5 \mathrm{D} \mathrm{U}(1)^{3}$ supergravity solution (see e.g. [6, 21-23]). It can be done by uplifting the solution to six dimensions, treating one of the gauge fields as a Kaluza-Klein one, and making a boost along the compact sixth direction. The other two gauge fields at the same time combine into the 2 -from. Then one can reduce the result back to five dimensions and repeat the procedure with the next gauge field. After three boosts one obtains the general solution with three independent electric charges. The charges are parametrized by the velocities $\beta_{i}$ of the boosts.

\section{Solution}

In this section we will present the general black ring solution of the $5 \mathrm{D} \mathrm{U}(1)^{3}$ supergravity field equations. The field equations themselves are written down in the appendix A. We will express the components of the fields in terms of a set of auxiliary functions defined in the appendix B. Let us start from the scalar fields $\Phi_{i}$. They have the following form:

$$
e^{\Phi_{i}}=\frac{\chi_{i}}{\chi}, \chi_{i}=H_{0 i}-\beta_{i}^{2} \tilde{H}_{0 i}+2 \beta_{i} K_{0 i}, \chi=\prod_{i=1}^{3} \chi_{i}^{1 / 3}
$$

The $t t$ component of the inverse metric is:

$$
g^{t t}=\chi^{-1}\left(\sum_{m, n=0}^{3}\left(-2 \beta_{0} \frac{\beta_{n}}{\beta_{m}} \Pi_{m n}-\beta_{0}^{2} \frac{\Xi_{m n}}{\beta_{m} \beta_{n}}\right)+\sum_{m=0}^{3}\left(\beta_{0}^{2} \frac{\Sigma_{m}}{\beta_{m}^{2}}-\beta_{m}^{2} \tilde{\Sigma}_{m}\right)+2 \beta_{0} Q\right)
$$

where the diagonal elements of $\Pi$ and $\Xi$ are defined to be zero: $\Pi_{m m}=\Xi_{m m}=0$ and a shorthand notation $\beta_{0}=-\beta_{1} \beta_{2} \beta_{3}$ is introduced. The other non-zero inverse metric 
components are:

$$
\begin{aligned}
g^{t \varphi}= & \chi^{-1} \sum_{m=0}^{3}\left(\beta_{m} \tilde{\omega}_{m}^{\psi}+\frac{\beta_{0}}{\beta_{m}} \omega_{m}^{\varphi}\right), \quad g^{\varphi \varphi}=\frac{Z}{\chi}, \quad g^{\psi \psi}=-\frac{\tilde{Z}}{\chi}, \\
g^{\varphi \psi}= & -\chi^{-1}\left\|a \frac{r(u, v)}{u-v} q\left(y_{0}\right) q\left(y_{1}\right) q\left(y_{2}\right) q\left(y_{3}\right) S^{\prime}\left\{\frac{(u-v)^{2}}{l(u) l(v)}\right\}\right\| \\
& +\frac{u-v}{\chi G(u) G(v)} \prod_{m=0}^{3} G\left(y_{m}\right) c_{3}\left(\frac{a r(u, v) \Delta}{q\left(y_{0}\right) q\left(y_{1}\right) q\left(y_{2}\right) q\left(y_{3}\right)}\right) . \\
g^{u u}= & \frac{(u-v)^{2}}{C_{0} \chi} G(u), \quad g^{v v}=-\frac{(u-v)^{2}}{C_{0} \chi} G(v),
\end{aligned}
$$

where $C_{0}$ is an arbitrary constant.

The determinant of the $3 \times 3$ matrix of the inverse metric components $g^{m n}(m, n=$ $t, \varphi, \psi)$ has the simple form:

$$
\operatorname{det}\left(g^{m n}\right)=\frac{(u-v)^{4}}{G(u) G(v)} .
$$

The gauge vector potentials are:

$$
\begin{aligned}
A_{t}^{i} & =\frac{1}{\chi_{i}}\left(\frac{1+\beta_{i}^{2}}{1-\beta_{i}^{2}} K_{0 i}+\frac{\beta_{i}}{1-\beta_{i}^{2}}\left(H_{0 i}-\tilde{H}_{0 i}\right)\right), \\
A_{\varphi}^{i} & =\frac{1}{\chi_{i}}\left(\Omega_{\varphi}^{i 0}-\beta_{j} \beta_{k} \Omega_{\varphi}^{0 i}+\beta_{i} \beta_{j} \Omega_{\varphi}^{j k}+\beta_{i} \beta_{k} \Omega_{\varphi}^{k j}+\beta_{1} \beta_{2} \beta_{3} \tilde{\Omega}_{\psi}^{i 0}-\beta_{i} \tilde{\Omega}_{\psi}^{0 i}+\beta_{k} \tilde{\Omega}_{\psi}^{j k}+\beta_{j} \tilde{\Omega}_{\psi}^{k j}\right) .
\end{aligned}
$$

$A_{\psi}^{i}$ can be obtained from $A_{\varphi}^{i}$ by exchanging all $\phi$ and $\psi$ subscripts in the expression above.

\section{Conclusions}

In this paper we presented the general black ring solution in $\mathrm{U}(1)^{3}$ supergravity (and therefore in the minimal 5D supergravity as well). We have tried to simplify it as much as possible. To this end we introduced several notations, which allowed to reduce the length of expressions considerably. Still we are not completely satisfied at this point with the form of the solution. One can hope that there is a formulation that is both elegant and allows to check the validity of the solution analytically, instead of checking it numerically, as we were forced to do. Such formulation would uncover the natural algebraic structure of the solution and give it a nice mathematical sense.

One possible way to reach such better understanding of the solution is to try to generalize it to a larger supergravity that reduces to the $\mathrm{U}(1)^{3}$ theory when some fields vanish. Interesting examples are 11D supergravity (the low energy limit of M-theory) reduced to $5 \mathrm{D}$ on $T^{6}$ or on $K 3 \times T^{2}$ which gives theories with 27 gauge fields [24, 25]. In the first case some of the missing dipole charges can be generated by suitably uplifting our solution to $11 \mathrm{D}$ and then rotating it in the six compact directions. Combining this rotations with duality transformations can probably generate even more independent dipole charges.

As an intermediate step one could also try to find the black ring solutions in the theory with just one additional gauge field considered in [23]. It would be straightforward to add 
an electric charge with respect to the additional gauge field. Adding the dipole charge using symmetry considerations is not straightforward, but may turn out to be possible with some luck. Another interesting problem is a deeper investigation of the black ring solution in minimal 5D supergravity. In this case the number of independent parameters and fields is smaller than in more general case of $\mathrm{U}(1)^{3}$ supergravity, and therefore one can hope to be able to get more explicit expressions for the regular solution and its mass, angular momenta etc.

\section{Acknowledgments}

A.F. acknowledges the financial support by the Dynasty Foundation.

\section{A Field equations}

In this appendix the Greek indices enumerate the five-dimensional spacetime coordinates, and the summation over repeating Greek indices is assumed. The field equations for the $\mathrm{U}(1)^{3}$ five-dimensional supergravity can be derived from the following action:

$$
I=\int d^{5} x \sqrt{-g}\left(R-\frac{1}{4} \sum_{i=1}^{3} e^{2 \Phi_{i}} F_{\mu \nu}^{i} F^{i \mu \nu}-\frac{1}{2} \sum_{i=1}^{3} g^{\mu \nu} \partial_{\mu} \Phi_{i} \partial_{\nu} \Phi_{i}\right)-\int d A^{1} \wedge d A^{2} \wedge A^{3},
$$

with the constraint $\Phi_{1}+\Phi_{2}+\Phi_{3}=0$ and where $F^{i}=d A^{i}$. The resulting field equations have the form

$$
\begin{aligned}
\partial_{\nu}\left(\sqrt{-g} e^{a \Phi_{i}} F^{i \sigma \nu}\right)= & \frac{1}{4} \epsilon^{\mu \nu \kappa \lambda \sigma} F_{\mu \nu}^{j} F_{\kappa \lambda}^{k} \\
\partial_{\mu}\left(\sqrt{-g} g^{\mu \nu} \partial_{\nu} \Phi_{i}\right)= & \frac{\sqrt{-g}}{6}\left(2 e^{2 \Phi_{i}} F_{\mu \nu}^{i} F^{i \mu \nu}-e^{2 \Phi_{j}} F_{\mu \nu}^{j} F^{j \mu \nu}-e^{2 \Phi_{k}} F_{\mu \nu}^{k} F^{k \mu \nu}\right) \\
R_{\mu \nu}-\frac{1}{2} g_{\mu \nu} R= & \frac{1}{2} \sum_{i=1}^{3} e^{2 \Phi_{i}}\left(F_{\lambda \mu}^{i} F_{\nu}^{i \lambda}-\frac{1}{4} g_{\mu \nu} F_{\kappa \lambda}^{i} F^{i \kappa \lambda}\right) \\
& +\frac{1}{2} \sum_{i=1}^{3}\left(\partial_{\mu} \Phi_{i} \partial_{\nu} \Phi_{i}-\frac{1}{2} g_{\mu \nu} g^{\kappa \lambda} \partial_{\kappa} \Phi_{i} \partial_{\lambda} \Phi_{i}\right)
\end{aligned}
$$

Let us write down the equations for the gauge field strength more explicitly:

$$
\begin{gathered}
\partial_{u}\left(\frac{G(u)}{(u-v)^{2}} e^{2 \Phi_{i}} \sum_{n=t, \varphi, \psi} g^{m n} \partial_{u} A_{n}^{i}-\sum_{n=t, \varphi, \psi} \sum_{s=t, \varphi, \psi} \epsilon^{m n s} A_{n}^{j} \partial_{v} A_{s}^{k}\right) \\
=\partial_{v}\left(\frac{G(v)}{(u-v)^{2}} e^{2 \Phi_{i}} \sum_{n=t, \varphi, \psi} g^{m n} \partial_{v} A_{n}^{i}-\sum_{n=t, \varphi, \psi} \sum_{s=t, \varphi, \psi} \epsilon^{m n s} A_{n}^{j} \partial_{u} A_{s}^{k}\right)
\end{gathered}
$$

where $\epsilon^{m n s}$ is the antisymmetric tensor and $\epsilon^{t \varphi \psi}=1$. The field equations for the dilatons can be reduced to the statement, that the expression

$$
\begin{aligned}
\partial_{u}\left(\frac{G(u)}{(u-v)^{2}} \partial_{u} \Phi_{i}\right)-\partial_{v}\left(\frac{G(v)}{(u-v)^{2}} \partial_{v} \Phi_{i}\right) \\
\quad-\sum_{m, n=t, \varphi, \psi} e^{2 \Phi_{i}}\left(\frac{G(u)}{(u-v)^{2}} g^{m n} \partial_{u} A_{m}^{i} \partial_{u} A_{n}^{i}-\frac{G(v)}{(u-v)^{2}} g^{m n} \partial_{v} A_{m}^{i} \partial_{v} A_{n}^{i}\right)
\end{aligned}
$$


does not depend on $i$ and the constraint $\Phi_{1}+\Phi_{2}+\Phi_{3}=0$ is satisfied. Finally, the Einstein equations can be reduced to the following form:

$$
\begin{aligned}
\partial_{u}\left(\frac{G(u)}{(u-v)^{2}} \sum_{s=t, \varphi, \psi} g_{m s} \partial_{u} g^{s n}\right)-\partial_{v}\left(\frac{G(v)}{(u-v)^{2}} \sum_{s=t, \varphi, \psi} g_{m s} \partial_{v} g^{s n}\right) \\
=\sum_{i=1}^{3} \sum_{s=t, \varphi, \psi} e^{2 \Phi_{i}}\left(\frac{G(u)}{(u-v)^{2}} \partial_{u} A_{m}^{i} g^{n s} \partial_{u} A_{s}^{i}-\frac{G(v)}{(u-v)^{2}} \partial_{v} A_{m}^{i} g^{n s} \partial_{v} A_{s}^{i}\right) \\
-\frac{1}{3} \delta_{m}^{n} \sum_{i=1}^{3} \sum_{p, s=t, \varphi, \psi} e^{2 \Phi_{i}}\left(\frac{G(u)}{(u-v)^{2}} \partial_{u} A_{p}^{i} g^{p s} \partial_{u} A_{s}^{i}-\frac{G(v)}{(u-v)^{2}} \partial_{v} A_{p}^{i} g^{p s} \partial_{v} A_{s}^{i}\right) .
\end{aligned}
$$

\section{B Set of functions}

In this appendix we define the set of function used in section 4 to write down the black ring solution. The notations used here were described in section 2 .

$$
\begin{aligned}
H_{i j}= & -S\left\{\frac{1}{(u-v)^{2}}\left(u-y_{i}\right)\left(u-y_{j}\right)\left(v-y_{k}\right)\left(v-y_{l}\right)\right\} \\
& \quad+\frac{1}{(u-v)^{2}} c\left(a r(u, v), a r(u, v), \Delta q\left(y_{i}\right) q\left(y_{j}\right) q\left(y_{k}\right) q\left(y_{l}\right)\right) \\
K_{i j}= & \left\|S^{\prime}\left\{\frac{a r(u, v)}{u-v}\right\} q\left(y_{i}\right) q\left(y_{j}\right) \bar{q}\left(y_{k}\right) \bar{q}\left(y_{l}\right)\right\| \\
\Omega_{\varphi}^{i j}= & \left\|\frac{a r(u, v)}{u-v} q\left(y_{i}\right) \bar{q}\left(y_{j}\right) \bar{q}\left(y_{k}\right) \bar{q}\left(y_{l}\right) S^{\prime}\left\{u-y_{j}-\frac{l(u)}{u-v}\right\}\right\| \\
\Omega_{\psi}^{i j}= & -\prod_{m=1}^{4} \sqrt{x_{m}-y_{i}} \frac{\partial H_{i j}}{\partial y_{i}}, \\
Z= & -S\left\{\frac{1}{G(v)}\left(v-y_{0}\right)\left(v-y_{1}\right)\left(v-y_{2}\right)\left(v-y_{3}\right)\right\} \\
& +c\left(a r(u, v), a r(u, v), \bar{\Delta} q\left(y_{0}\right) q\left(y_{1}\right) q\left(y_{2}\right) q\left(y_{3}\right)\left(\frac{1}{\bar{l}(u) l(v)}-\frac{a^{2}}{l(u) \bar{l}(v)}\right)\right) \\
\omega_{i}^{\varphi}= & \prod_{m=1}^{4} \sqrt{x_{m}-y_{i}} \frac{\partial Z}{\partial y_{i}}, \\
\omega_{i}^{\psi}= & \left\|\frac{a r(u, v)}{u-v} \bar{q}\left(y_{i}\right) q\left(y_{j}\right) q\left(y_{k}\right) q\left(y_{l}\right) S^{\prime}\left\{\frac{u-v}{l(v)}\left(1-\frac{u-v}{l(u)}\left(u-y_{i}\right)\right)\right\}\right\| \\
G(u) G(v) & \prod_{m=1} \sqrt{x_{m}-y_{i}} G\left(y_{j}\right) G\left(y_{k}\right) G\left(y_{l}\right) c_{3}\left(\frac{a r(u, v) \Delta}{q\left(y_{j}\right) q\left(y_{k}\right) q\left(y_{l}\right)}\right)
\end{aligned}
$$




$$
\begin{aligned}
& \Sigma_{i}=S\left\{\left(\frac{\left(u-y_{i}\right)^{2}}{(u-v)^{2}}-\frac{G\left(y_{i}\right)}{G(v)}\right) \frac{\left(v-y_{j}\right)\left(v-y_{k}\right)\left(v-y_{l}\right)}{\left(v-y_{i}\right)}\right\} \\
& -c\left(a r(u, v), a r(u, v), \Delta q\left(y_{1}\right) q\left(y_{2}\right) q\left(y_{3}\right) q\left(y_{4}\right) \times\right. \\
& \left.\times\left(\frac{1-a^{2}}{(u-v)^{2}}-\bar{l}\left(y_{i}\right)\left(\frac{1}{\bar{l}(u) l(v)}-\frac{a^{2}}{l(u) \bar{l}(v)}\right)\right)\right), \\
& \Pi_{i j}=\left\|\frac{a r(u, v)}{u-v} \bar{q}\left(y_{i}\right) \bar{q}\left(y_{j}\right) q\left(y_{k}\right) q\left(y_{l}\right) S^{\prime}\left\{\left(1-\frac{u-v}{l(u)}\left(u-y_{i}\right)\right)\left(1-\frac{v-u}{l(v)}\left(v-y_{j}\right)\right)\right\}\right\| \\
& +\frac{u-v}{G(u) G(v)} G\left(y_{k}\right) G\left(y_{l}\right) \prod_{m=1}^{4} \sqrt{x_{m}-y_{i}} \sqrt{x_{m}-y_{j}} c_{3}\left(\frac{a r(u, v) \Delta}{q\left(y_{k}\right) q\left(y_{l}\right)}\right), \\
& \Xi_{i j}=-\prod_{m=1}^{4} \sqrt{x_{m}-y_{i}} \sqrt{x_{m}-y_{j}} \frac{\partial^{2} Z}{\partial y_{i} \partial y_{j}}-\prod_{m=1}^{4} \sqrt{x_{m}-y_{k}} \sqrt{x_{m}-y_{l}} \frac{\partial^{2} Z}{\partial y_{k} \partial y_{l}} \\
& +\frac{1}{2} c\left(a r(u, v), a r(u, v), \Delta \bar{\Delta} q\left(y_{i}\right) q\left(y_{j}\right) \bar{q}\left(y_{k}\right) \bar{q}\left(y_{l}\right)\left(\frac{1}{\bar{l}(u) l(v)}-\frac{a^{2}}{l(u) \bar{l}(v)}\right)\right), \\
& Q=\left\|a \frac{r(u, v)}{u-v} q\left(y_{0}\right) q\left(y_{1}\right) q\left(y_{2}\right) q\left(y_{3}\right)\left(\bar{l}\left(y_{0}\right)+\bar{l}\left(y_{1}\right)+\bar{l}\left(y_{2}\right)+\bar{l}\left(y_{3}\right)\right) S^{\prime}\left\{\frac{(u-v)^{2}}{l(u) l(v)}\right\}\right\| \\
& -\frac{u-v}{G(u) G(v)} \prod_{m=0}^{3} G\left(y_{m}\right) \sum_{n=0}^{3} \frac{1}{G\left(y_{n}\right)} c_{3}\left(\frac{a r(u, v) q\left(y_{n}\right) \Delta}{q\left(y_{0}\right) q\left(y_{1}\right) q\left(y_{2}\right) q\left(y_{3}\right)}\right) .
\end{aligned}
$$

Open Access. This article is distributed under the terms of the Creative Commons Attribution License (CC-BY 4.0), which permits any use, distribution and reproduction in any medium, provided the original author(s) and source are credited.

\section{References}

[1] R. Emparan and H.S. Reall, Black rings, Class. Quant. Grav. 23 (2006) R169 [hep-th/0608012] [INSPIRE].

[2] R. Emparan and H.S. Reall, A rotating black ring solution in five-dimensions, Phys. Rev. Lett. 88 (2002) 101101 [hep-th/0110260] [INSPIRE].

[3] H. Elvang, A charged rotating black ring, Phys. Rev. D 68 (2003) 124016 [hep-th/0305247] [INSPIRE].

[4] H. Elvang and R.a. Emparan, Black rings, supertubes and a stringy resolution of black hole nonuniqueness, JHEP 11 (2003) 035 [hep-th/0310008] [INSPIRE].

[5] R. Emparan, Rotating circular strings and infinite nonuniqueness of black rings, JHEP 03 (2004) 064 [hep-th/0402149] [INSPIRE].

[6] H. Elvang, R. Emparan and P. Figueras, Non-supersymmetric black rings as thermally excited supertubes, JHEP 02 (2005) 031 [hep-th/0412130] [INSPIRE].

[7] H. Elvang, R. Emparan, D. Mateos and H.S. Reall, A Supersymmetric black ring, Phys. Rev. Lett. 93 (2004) 211302 [hep-th/0407065] [INSPIRE]. 
[8] I. Bena and N.P. Warner, One ring to rule them all ... and in the darkness bind them?, Adv. Theor. Math. Phys. 9 (2005) 667 [hep-th/0408106] [INSPIRE].

[9] H. Elvang, R. Emparan, D. Mateos and H.S. Reall, Supersymmetric black rings and three-charge supertubes, Phys. Rev. D 71 (2005) 024033 [hep-th/0408120] [INSPIRE].

[10] J.P. Gauntlett and J.B. Gutowski, General concentric black rings, Phys. Rev. D 71 (2005) 045002 [hep-th/0408122] [INSPIRE].

[11] Y. Chen, K. Hong and E. Teo, A doubly rotating black ring with dipole charge, JHEP 06 (2012) 148 [arXiv: 1204.5785] [InSPIRE].

[12] J.V. Rocha, M.J. Rodriguez and O. Varela, An electrically charged doubly spinning dipole black ring, JHEP 12 (2012) 121 [arXiv:1205.0527] [INSPIRE].

[13] A. Feldman and A.A. Pomeransky, Charged black rings in supergravity with a single non-zero gauge field, JHEP 07 (2012) 141 [arXiv:1206.1026] [INSPIRE].

[14] V.A. Belinsky and V.E. Zakharov, Integration of the Einstein equations by the inverse scattering problem technique and the calculation of the exact soliton solutions, Sov. Phys. JETP 48 (1978) 985 [INSPIRE].

[15] V.A. Belinsky and V.E. Sakharov, Stationary gravitational solitons with axial symmetry, Sov. Phys. JETP 50 (1979) 1 [INSPIRE].

[16] V. Belinski and E. Verdaguer, Gravitational solitons, Cambridge University Press, Cambridge U.K. (2005).

[17] J.V. Rocha, M.J. Rodriguez and A. Virmani, Inverse scattering construction of a dipole black ring, JHEP 11 (2011) 008 [arXiv:1108.3527] [INSPIRE].

[18] A.A. Pomeransky, Complete integrability of higher-dimensional Einstein equations with additional symmetry and rotating black holes, Phys. Rev. D 73 (2006) 044004 [hep-th/0507250] [INSPIRE].

[19] A.A. Pomeransky and R.A. Sen'kov, Black ring with two angular momenta, hep-th/0612005 [INSPIRE].

[20] T. Harmark, Stationary and axisymmetric solutions of higher-dimensional general relativity, Phys. Rev. D 70 (2004) 124002 [hep-th/0408141] [INSPIRE].

[21] J. Hoskisson, A charged doubly spinning black ring, Phys. Rev. D 79 (2009) 104022 [arXiv:0808.3000] [INSPIRE].

[22] D.V. Gal'tsov and N.G. Scherbluk, Three-charge doubly rotating black ring, Phys. Rev. D 81 (2010) 044028 [arXiv:0912.2771] [INSPIRE].

[23] J.C. Breckenridge, R.C. Myers, A.W. Peet and C. Vafa, D-branes and spinning black holes, Phys. Lett. B 391 (1997) 93 [hep-th/9602065] [INSPIRE].

[24] R. Emparan and H.S. Reall, Black holes in higher dimensions, Living Rev. Rel. 11 (2008) 6 [arXiv:0801.3471] [INSPIRE].

[25] M. Cvetič and D. Youm, General rotating five-dimensional black holes of toroidally compactified heterotic string, Nucl. Phys. B 476 (1996) 118 [hep-th/9603100] [INSPIRE]. 